rev.relac.int.estrateg.segur.7(2):53-74,2012

\title{
TIMOR ORIENTAL: INTERVENCIÓN INTERNACIONAL Y DELIMITACIÓN MARÍTIMA*
}

\author{
Camilo Andrés Devia Garzón** \\ Walter René Cadena Afanador ${ }^{* *}$
}

\section{RESUMEN}

La intervención internacional en el conflicto del Timor Oriental se presenta como una Operación de Mantenimiento de Paz paradigmática. Aunque la contribución de Australia fue vital para garantizar el éxito de esta operación, su participación también respondió a otros intereses como la delimitación marítima en condiciones favorables para Australia y la

* Este artículo de investigación es resultado del proyecto EES 927 "La Convención de Naciones Unidas del Derecho del Mar (1982) y su no ratificación por parte de Colombia" financiado por la Universidad Militar Nueva Granada (UMNG) y adscrito a las Facultades de Derecho, y de Relaciones Internacionales, Estrategia y Seguridad (Faries). Se destaca la colaboración en este proyecto de siete estudiantes investigadores de las dos Facultades. Este trabajo recoge algunas reflexiones presentadas durante las $<<$ IV Jornadas de Estudios de Seguridad $>>$ organizadas por el Instituto Universitario <<General Gutiérrez Mellado >> (IUGM - UNED), Madrid.

** Profesional en Relaciones Internacionales y Estudios Políticos (UMNG), Estudiante de la maestría en Asuntos Internacionales (Universidad Externado de Colombia). Docente investigador, Faries - UMNG. Correo electrónico: camilo.devia@unimilitar.edu.co.

*** Abogado (UNAB), Magíster en Relaciones Internacionales (Pontificia Universidad Javeriana). Estudiante del Doctorado en Derecho (Universidad de Buenos Aires). Docente investigador, Derecho - UMNG y Universidad Libre. Correo electrónico: walter.cadena@unimilitar.edu.co 
explotación de las reservas petrolíferas ubicadas en el Mar de Timor. Estas acciones legitimadas por la comunidad internacional han contribuido a debilitar la construcción de la paz y reavivar el conflicto armado.

Palabras clave: Timor Leste, Australia, Delimitación marítima, Operación de Mantenimiento de Paz.

\title{
EAST TIMOR: \\ INTERNATIONAL INTERVENTION \\ AND MARITIME DEMARCATION
}

\begin{abstract}
The international intervention in the East Timor conflict presents itself as a paradigmatic Peacekeeping Operation. While Australia's contribution was fundamental to guarantee the success of this operation, its participation also answered to other interests like a maritime demarcation favorable to Australia and the exploration of oil reserves in the Timor Sea. These actions, legitimated by the international community, have contributed to the weakening of peace and the revival of the armed conflict.
\end{abstract}

Key words: East Timor, Australia, Maritime Demarcation, Peacekeeping Operation.

\section{TIMOR-LESTE: \\ INTERVENÇÃO INTERNACIONAL \\ E DELIMITAÇÃO MARÍTIMA}

\section{RESUMO}

A Intervenção internacional no conflito do Timor-Leste apresenta-se como uma Operação de Manutenção de Paz paradigmática. Embora a contribuição da Austrália fosse fundamental para garantir o sucesso desta operação, sua participação também respondeu a outros interesses como a delimitação marítima que beneficiou a Austrália e a exploração das reservas de petróleo localizadas no Mar do Timor. Essas ações legitimadas pela comunidade internacional têm contribuído para o enfraquecimento da construção da paz e para reacender o conflito armado.

Palavras-chave: Timor-leste, Austrália, Delimitação marítima, Operação de Manutenção da Paz. 


\section{INTRODUCCIÓN}

La intervención de las Naciones Unidas ha sido determinante para la historia reciente de Timor Leste. Después de un largo y complejo proceso de lucha por la autodeterminación, solo con el establecimiento de una Operación de Mantenimiento de Paz fue posible la instauración del Estado independiente y la estabilización de la preocupante situación de violencia que se desató en el territorio.

Australia, potencia media en el sudeste asiático ${ }^{1}$, tuvo una participación clave en la intervención de Naciones Unidas en Timor. En cada una de las diferentes misiones que constituyen los 13 años de tareas en Timor, el liderazgo de Australia es destacado como la punta de lanza de una compleja tarea humanitaria.

Sin embargo, se pretende mostrar como lejos del interés altruista la participación australiana responde al interés económico relacionado con la explotación de los importantes recursos de hidrocarburos presentes en el Mar de Timor. La cuestión radica en que no está definido el límite marítimo entre Australia y Timor, ya que Portugal como colonizador, ni Indonesia en el momento de la anexión, se sometieron a las pretensiones australianas, lejos de lo dispuesto en la Convención del Derecho del Mar de 1982.

\section{EL CONFLICTO EN TIMOR LESTE}

El conflicto de Timor Leste se remonta al periodo de la colonización. En el marco de los procesos de expansión de las potencias colonizadoras europeas en el siglo XVI, Holanda y Portugal arribaron a la isla de Timor y tras algunos enfrentamientos iniciales se estableció la repartición de la isla en dos partes.

El interés de Portugal sobre el territorio oriental de la isla de Timor fue siempre marginal. La gran distancia existente entra la isla y Portugal y la escasa importancia en cuanto a riquezas naturales, hicieron que no existiera una política para generar desarrollo de infraestructura o

1 "Las potencias medias son Estados centrales y desarrollados, modernos, democráticos y con una diplomacia muy especializada de alcance global. Estas potencias muestran indicadores elevados de capacidades inmateriales. No obstante, a diferencia de las potencias mundiales, las potencias medias cuentan con capacidades materiales más limitadas, restricción que las ubica jerárquicamente por debajo de las potencias mundiales, pero por encima de las potencias regionales. Es importante anotar que estas potencias medias difícilmente lograrán ampliar sus capacidades materiales, pues este es su talón de Aquiles. Alno ser potencias mundiales, no están en el G7 ni en el Consejo de Seguridad, pero sí son miembros de la OCDE y juegan papeles importantes en las instituciones y regímenes internacionales. En esta categoría se encuentran: Australia, Holanda, España, Dinamarca, Noruega, Corea del Sur, Suecia, Israel y Bélgica" (Rocha \& Morales, 2010). 
de instituciones de gobierno. Además, Portugal estimuló las rivalidades entre las diferentes tribus como forma para impedir los levantamientos (CAVR, 2005, Parte 3, 2).

La descolonización, llevada a cabo en agosto de 1975, se caracterizó entonces por ser producto de procesos internos en el país colonizador y no como un resultado directo de la lucha del pueblo de Timor Oriental por su autodeterminación. Sin embargo, una semana después de la fecha de retirada de los portugueses, Indonesia ocupó militarmente el territorio y la convirtió en su provincia número 27. La anexión fue apoyada por Estados Unidos y Australia bajo la justificación de la lucha contra el comunismo en el marco de la Guerra Fría.

Las iniciativas del gobierno de Yakarta se centraron en la consolidación del dominio territorial a través del ejercicio de la fuerza y de diversas campañas militares (CAVR, 2005, Parte 3, p. 223-376; Taylor, 2001, p. 216). Lejos de desarrollar un proceso de asimilación cultural, social y étnico de la población timorense, la brutal represión militar provocó el efecto inverso. La Comisión para la Acogida, la Verdad y la Reconciliación (CAVR) estima que en el periodo de la anexión indonesia de Timor, entre 1975 y 1999, el número de muertes relacionadas con el conflicto fueron cerca de 102.800. Además, la Comisión informa sobre un estimado de 282.800 desplazados, en su mayoría internos, esto es, más de una cuarta parte de la población nacional.

La última década del siglo XX presentó acontecimientos que facilitaron la exposición internacional de la violencia desplegada en Timor Oriental, permitiendo una sensibilización de la sociedad internacional frente al inconcluso proceso de descolonización. Ello condujo a un paulatino proceso de independencia, el cual fue tutelado por las Naciones Unidas y que se materializó con la aprobación y despliegue de Operaciones de Mantenimiento de Paz en el territorio insular. Entre los acontecimientos de impacto internacional se destacan los siguientes:

a) Visita de Juan Pablo II a Dili, capital de Timor Oriental, en octubre de 1989. La visita fue de carácter evangélico, ya que el 77\% de la población timorense de entonces profesaba la religión católica ${ }^{2}$. El gobierno indonesio trató de interpretar la visita como un reconocimiento por parte del Vaticano a la anexión indonesia de Timor Oriental. Sin embargo, el Papa evitó hacer referencias directas a esta situación. Por ejemplo, al llegar

2. La labor de Monseñor Martinho Costa Lopes y, posteriormente, del Obispo Carlos Filipe Ximenes Belo, permitieron que la labor de la Iglesia Católica durante el conflicto no fuese contemplativa sino que fuese activa en denunciar los abusos cometidos por las tropas indonesias. Los timorenses percibieron, después de la invasión, que los jerarcas de la religión católica eran solidarios con su problemática y les brindaban protección, bajo un sentido de santuario y supervivencia. De allí que no sea coincidencia que para 1973 sólo el 28\% de la población era católica, mientras que en 1980, más del 80\% de la población se había convertido al catolicismo. Por su labor, el Obispo Ximenes Belo sería galardonado con el Premio Nobel de Paz (CAVR, 2005, Parte 3, p. 397-399). 
al aeropuerto, el Papa no besó la tierra (signo de independencia timorense), pero tampoco ignoró este acto (que sería valorado como un reconocimiento de la soberanía legal de Indonesia sobre la isla). En su lugar besó un crucifijo colocado sobre la tierra (Guardiola, 1989).

b) La masacre del Cementerio de Santa Cruz, ocurrida el 12 de noviembre de 1991. Sucedió después del asesinato de Sebastião Gomes, uno de los cuadros del Fretilin. Durante las exequias de Gomes y, aprovechando la llegada de un delegado especial de las Naciones Unidas, se realizó una demostración masiva de rechazo a este asesinato, que transcurrió de manera pacífica, pero que posteriormente acabó en un baño de sangre. Se estima que fueron aproximadamente 200 muertos (CAVR, Parte 3, p. 475-486).

c) La captura de José Alexandre XananaGusmão, un importante líder de la resistencia timorense, en noviembre de 1992. La captura de Gusmão fue el más importante golpe que Indonesia le propinó a la resistencia timorense, pero a la postre lo convirtió en un ícono que le reportó visibilidad y apoyo internacional a la causas de la autodeterminación timorense. Xanana permaneció recluido en la prisión de alta seguridad de Cipinang, en Yakarta (CAVR, Parte 3, p. 487-488). Posteriormente, sería elegido como el primer presidente de Timor-Leste.

d) El otorgamiento del Premio Nobel de la Paz al Obispo Carlos Filipe Ximenes Belo y a José Ramos-Horta en diciembre de 1996. En el discurso de presentación de estos premios, se señaló que:

(...) el conflicto en Timor Oriental ha sido llamado $<<$ el conflicto olvidado $>>$ (...). Rara vez el cinismo de la política mundial había sido más claro (...). Este año dos premios Nobel de la Paz, Carlos Filipe Xiemnes Belo y José Ramos-Horta, han trabajado incansablemente y con gran sacrificio personal, por su pueblo oprimido. En condiciones sumamente difíciles, han preservado su humanidad y la fe en el futuro (CAVR, Parte 3, p. 502-506).

Sin embargo, la consecución de la autodeterminación no ha sido el fin de los problemas. La instauración de un gobierno autónomo y el fin de la violencia indonesia, promovieron la erupción de problemáticas sociales. Ahora la violencia ha combinado un profundo descontento social por la difícil situación económica, problemas de discriminación entre grupos sociales y étnico-lingüísticos, y la aparición del crimen organizado. Bajo la ocupación, la identidad de la población se construyó en torno a la búsqueda de la independencia y la creación del Estado de Timor-Leste (Canas, 2009, p. 23). Sin embargo, la concreción del sueño independentista sacó a la luz los bajos niveles de cohesión y la debilidad de un proyecto homogéneo de construcción estatal. 


\section{LA INTERVENCIÓN INTERNACIONAL PARA TIMOR LESTE}

La intervención de las Naciones Unidas (NU) en Timor es catalogada como una de las misiones exitosas en el marco de las Operaciones de Mantenimiento de Paz. Se destaca como un hito en el proceso de intervención de las NU, en donde se pasó de las operaciones tradicionales de peaceenforcemet, hacia un nuevo esquema de intervención multidimensional (esto es, que incluyen a civiles, policías y militares), donde la misión se transformaba periódicamente para responder a las necesidades del Estado en construcción y que asumió la administración general del país.

Las Operaciones Multidimensionales es una evolución en el formato tradicional con que se desarrollaron las OMP, donde se establecen que los factores que deben ser tenidos en cuenta no son solamente militares o policiales, sino que también son de carácter estructural que permiten la estabilidad o viabilidad de un Estado dentro de la sociedad internacional. Como indica Zurbriggen:

La seguridad es hoy un concepto que abarca un amplio espectro de problemas económicos, sociales y políticos que, junto a los hechos del 2001 en Estados Unidos, han contribuido a una mayor seguritización de la agenda. Mientras en el norte las amenazas principales son el terrorismo, fundamentalismo religioso, crimen organizado -cuya novedad no es sólo la amenaza en sí, sino más bien su modo de funcionamiento y de articulación con el mundo globalizado el sur se ve afectado por la pobreza que erosiona la cohesión social y vulnera la estabilidad política de los Estados, como Haití y recientemente Bolivia. Podemos observar problemas que exceden parámetros comunes, convirtiéndose en multidimensionales. Desde luego que los problemas socio-económicos se pueden convertir en problemas de seguridad, pero también es claro que la mejor solución no es encararlos como un problema militar sin resolver las causas que los generan (2005, pp. 105-106).

En la primera etapa de las OMP multidimensionales en Timor Oriental tuvo un éxito indiscutible, con la realización del referéndum de autodeterminación y la estabilización del país luego de la violencia surgida con los resultados. Sin embargo, los problemas surgen con la creación de las condiciones para regresar el poder a la población local y con el resurgimiento de la violencia con la crisis de 2006.

\subsection{UNAMET - INTERFET}

La Misión (de Asistencia) de las Naciones Unidas en Timor Oriental (UNAMET) fue establecida mediante la Resolución 1246 del Consejo de Seguridad y estuvo en funciones entre junio y octubre de 1999, con un mandato claramente establecido: "organizar y llevar a cabo una consulta popular para determinar si el pueblo de Timor Oriental aceptaba una autonomía espe-

TIMOR ORIENTAL: INTERVENCIÓN INTERNACIONAL Y DELIMITACIÓN MARÍTIMA 
cial dentro de Indonesia o si, por el contrario, rechazaba esta propuesta, lo cual llevaría a la separación de esta región" (UNPeacekeeping, 2012).

UNAMET se instituyó entonces como una misión únicamente de elementos políticos que debía asegurar las condiciones políticas para el referéndum, los temas de registro y conteo de votos, labor que logro cumplir. Sin embargo, luego del referéndum del 30 de agosto de 1999 iniciaron una larga serie de enfrentamientos armados. Las represalias de las tropas indonesias y las milicias pro indonesias ocurridas después de conocidos los resultados del referéndum de 1999, dejaron aproximadamente 1.500 muertos y la mitad de la población desplazada, esto es, cerca de 550.000 personas. Así mismo, fue destruida el $70 \%$ de la infraestructura y el $100 \%$ de la red eléctrica del país (CAVR, 2005; Kingsbury, 2007).

En medio del caos y por las características de UNAMET, las Naciones Unidas se vieron en la necesidad de complementar la actuación de UNAMET con una fuerza armada multinacional. La Fuerza Internacional para Timor Oriental (INTERFET) entra en funciones en septiembre de 1999 y se mantuvo activa hasta febrero de 2000. INTERFET cumplió con su función de estabilizar el país, y promover la salida definitiva de las tropas indonesias del territorio de Timor Oriental, lo que significó el inicio del periodo de transición hacia la instauración del Estado de Timor Leste.

\subsection{UNTAET}

Ya con la decisión de la independencia expresada en las urnas por el pueblo timorense el proceso de construcción de la infraestructura estatal da inicio. Sin embargo, la preocupación por recaer en la violencia y la necesidad de apoyar el establecimiento de la institucionalidad democrática, llevó a las Naciones Unidas a establecer una nueva misión de paz con unas características sin antecedentes en los procesos de intervención.

La Administración de Transición de las Naciones Unidas en Timor Oriental (UNTAET) inicia el 25 de octubre de 1999, día en que terminó la gestión de UNAMET. Esta misión contaba con un mandato mucho más amplio, que entre sus tareas preveía la provisión de la seguridad, el mantenimiento de la ley y el orden, la provisión de los servicios sociales y promover el desarrollo (Butler, 2012). La UNTAET asumió la administración del territorio y del gobierno mientras se establecían las condiciones para entregar el poder a la población local, lo que también estaba entre las tareas de la misión.

Por esa amplitud de mandato, autores como Butler (2012) son muy críticos de la gestión de UNAMET, destacando que se concentró en los temas de seguridad y se descuidó la administración civil y la preparación para el gobierno autónomo, sumando a todo ello las tensiones burocráticas al interior de las Naciones Unidas sobre la planificación general de la operación. Además, la misión estuvo altamente centralizada en la capital. Las instituciones creadas mostraron un 
alcance muy limitado y su presencia, fuera de la capital, era mínima, circunstancia que condujo a que la mayoría de la población las percibiera de una manera distante y sin identificarse con ellas.

La estrategia de $<<$ timorización $>>$ emprendida por UNAMET no fue exitosa. La integración de los líderes locales en el proceso de transición no fue amplia y discriminó importantes sectores políticos. El año 2000 fue realmente difícil, ya que las condiciones económicas seguían empeorando (el desempleo llega al 80\%), y el descontento sobre la gestión de las Naciones Unidas se hacía generalizado (Funaki, 2009).

Con la necesidad de transferir rápidamente el poder, UNAMET convoca a la Asamblea Constituyente que redacta la Constitución para marzo de 2002, celebra las primeras elecciones parlamentarias en Timor Leste, con triunfo de Fretilin, y termina la transferencia con la elección presidencial de abril de 2002, con el triunfo de XananaGusmao.

\subsection{UNMISET - UNOTIL}

La compleja tarea que UNTAET intentó desarrollar en Timor Leste terminó en mayo de 2002, y fue inmediatamente reemplazado por una nueva misión. En aquella ocasión la Misión de Apoyo de las Naciones Unidas en Timor Oriental (UNMISET) fue establecida con un mandato más limitado. Después de entregar la administración a las elegidas autoridades políticas, UNMISET debía "prestar apoyo a la recién estrenada independencia de Timor Oriental hasta que todas las responsabilidades operativas fuesen transferidas a las autoridades de la nueva nación, denominada Timor-Leste, y para permitir que esta lograra la autosuficiencia" (UNPeacekeeping, 2012).

La misión fue una muestra de la intención de las Naciones Unidas de una salida progresiva, con disminución de responsabilidades en Timor Leste; una misión planteada para trabajar con el gobierno para garantizar el orden y la seguridad pública, y ayudar a en el desarrollo de una fuerza de policía nacional. Con una duración inicial de 12 meses, el Consejo de Seguridad decidió su renovación por dos periodos adicionales hasta mayo de 2005, aunque reduciendo su mandato ala prestación de apoyo a la administración pública y el sistema de justicia, al desarrollo de la aplicación del derecho y para garantizar la seguridad y la estabilidad (Alldén \& Amner, 2007).

Con unas condiciones que parecían ir en mejora para mayo de 2005, Naciones Unidas decidió no prorrogar el mandato de UNMISET y establecer una misión de características políticas que buscara la finalización del proceso de construcción de la paz. La Oficina de las Naciones Unidas en Timor-Leste (UNOTIL), que estuvo operativa desde mayo de 2005 hasta agosto de 2006, apoyó el desarrollo de importantes instituciones del Estado y de la policía, y proporcionó capacitación sobre la observancia de la gobernanza democrática y los derechos humanos (UNPeacekeeping, 2012). 
UNOTIL estaba programada para terminar su gestión en febrero de 2006 y dar plena autonomía al gobierno timorense. Sin embargo, la $<<$ crisis de los solicitantes ${ }^{3}>>$ y el enfrentamiento violento que generó, hizo que se mantuviera la oficina hasta agosto y que fuera sustituida por una nueva misión de mantenimiento de paz, que significó un retroceso en el proceso que llevaba la presencia de las Naciones Unidas.

\subsection{UNMIT}

En junio de 2006, debido a las difíciles condiciones de seguridad, las autoridades políticas de Timor Leste solicitaron al Secretario General de Naciones Unidas el establecimiento de fuerzas policiales de las Naciones Unidas en Timor-Leste, para mantener el orden público hasta que la policía nacional pudiese garantizar la reorganización y la reestructuración.

Apoyados en los informes de los enviados, del 25 de agosto de 2006, se estableció la Misión Integrada de las Naciones Unidas en Timor-Leste (UNMIT), para ayudar a superar las consecuencias de la crisis. El papel de la UNMIT se centró en la realización de algunos de los más básicas funciones del Estado, en particular los relacionados con el ámbito de la seguridad (UNPeacekeeping, 2012)

La puesta en actividades de UNMIT apoyó la mejora general en la situación de seguridad, al propiciar la realización de las elecciones parlamentarias y presidenciales de 2007 en un ambiente de calma. Como resultado de este proceso electoral fue elegido como presidente el ex Primer Ministro José Ramos-Horta. Los atentados de 2008 contra el presidente y el primer ministro pusieron en desafío la capacidad de la misión, pero fueron rápidamente controlados por las fuerzas multinacionales desplegadas por NU.

El mandato de UNMIT fue prolongado por el Consejo de Seguridad debido a la preocupación razonable acerca de que la salida del personal armado de la fuerza de las Naciones Unidas podría derivar en nuevo focos de violencia. Buscando fortalecer la institucionalidad política, se estableció la finalización de la misión para después de las elecciones presidenciales de abril de 2012 y la transferencia de mando entre el nuevo gobierno y el gobierno saliente.

Las elecciones fueron ganadas por José María Vasconcelos, más conocido como $<<$ Taur Matan Ruak $>>$, ex guerrillero del Fretilin, quien vencióal ex presidente del Parlamento Francisco

3. La $<<$ crisis de los solicitantes $>>$ "en enero de 2006, cuando un grupo de 159 soldados firmaron un documento en el que protestaban por el trato desigual y discriminatorio al interior del Ejército. Para marzo del mismo año, 600 soldados (denominados como "Los solicitantes"), en su mayoría originarios de la parte oeste del país, desertaron de la institución. La situación se desencadenó a partir delas tensiones entre el Ejército (F-FDTL) y la Policía (PNTL), en donde ésta última apoyó a "Los solicitantes", estimulando cruentos enfrentamientos y revueltas. El desorden originado sólo pudo ser contenido con la presencia de las fuerzas internacionales de paz y la renuncia del primer ministro Alkatiri, acusado de distribución de armas entre la población civil" (Cadena \&Devia, 2010, p. 62). 
Guterres, y el Premio Nobel de la Paz, José Ramos-Horta, quien perdió en la primera ronda (AFP, 2012). La entrega de poderes se realizó en mayo; sin embargo, el Consejo de Seguridad de NU consideró prorrogar nuevamente el mandato de UNMIT hasta diciembre de 2012. El nuevo presidente recibió en septiembre de 2012 la visita de la Secretaria de Estado estadounidense Hillary Clinton, lo que se constituyó en un fuerte espaldarazo al proceso de construcción estatal de esta joven nación.

\section{LA DELIMITACIÓN DE LA FRONTERA MARÍTIMA ENTRE TIMOR-LESTE Y AUSTRALIA}

\subsection{LA PARTICIPACIÓN DE AUSTRALIA EN LAS OPERACIONES DE MANTENIMIENTO DE PAZ EN TIMOR LESTE}

La participación de Australia en las diferentes Operaciones de Mantenimiento de Paz (OMP), desplegadas por las Naciones Unidas en Timor, es destacable desdela aprobación de su primer mandato. A partir de 1999 la presencia de personal australiano se justificó como respuesta a la emergencia humanitaria; sin embargo, intereses distintos pueden haber motivado tan activa participación (ver gráficos 1 y 2).

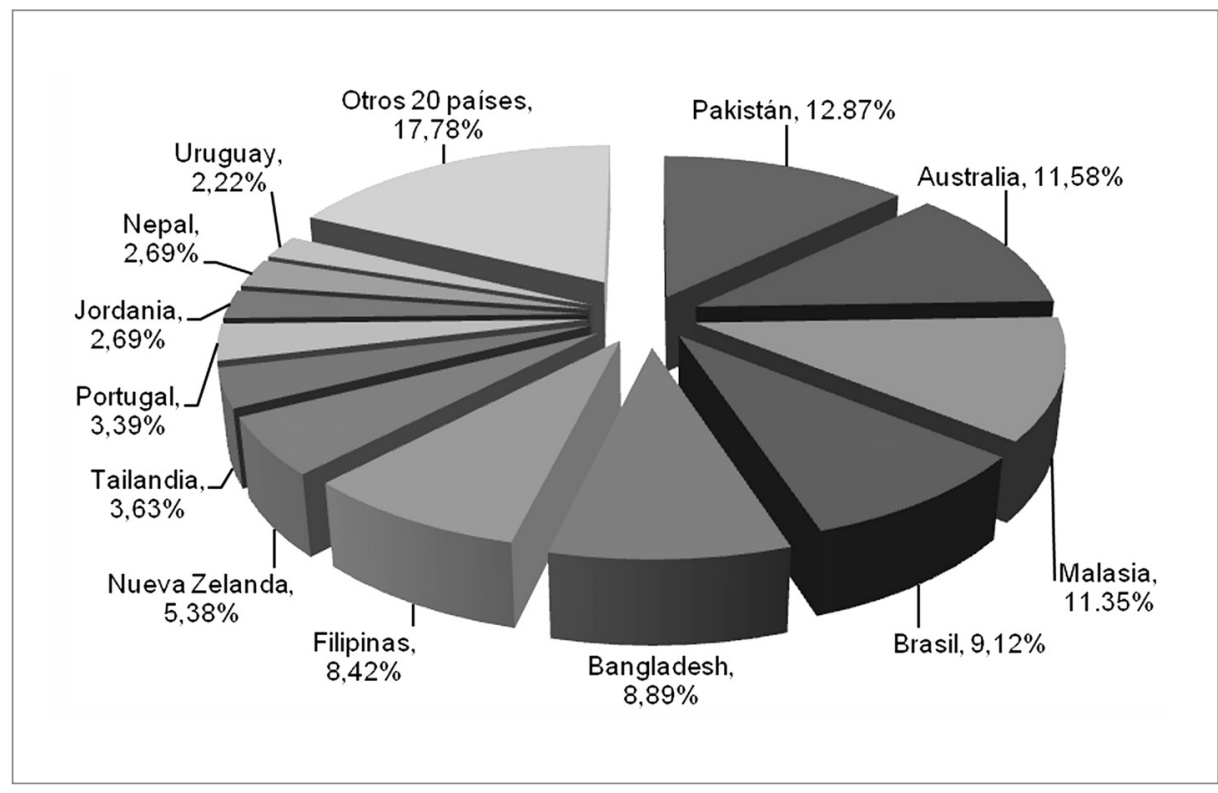

Gráfico 1: Porcentaje de observadores por país aportador en las diferentes misiones en Timor, 1999-2011

Fuente: elaboración propia a partir de los reportes mensuales de las NU sobre OMP. 


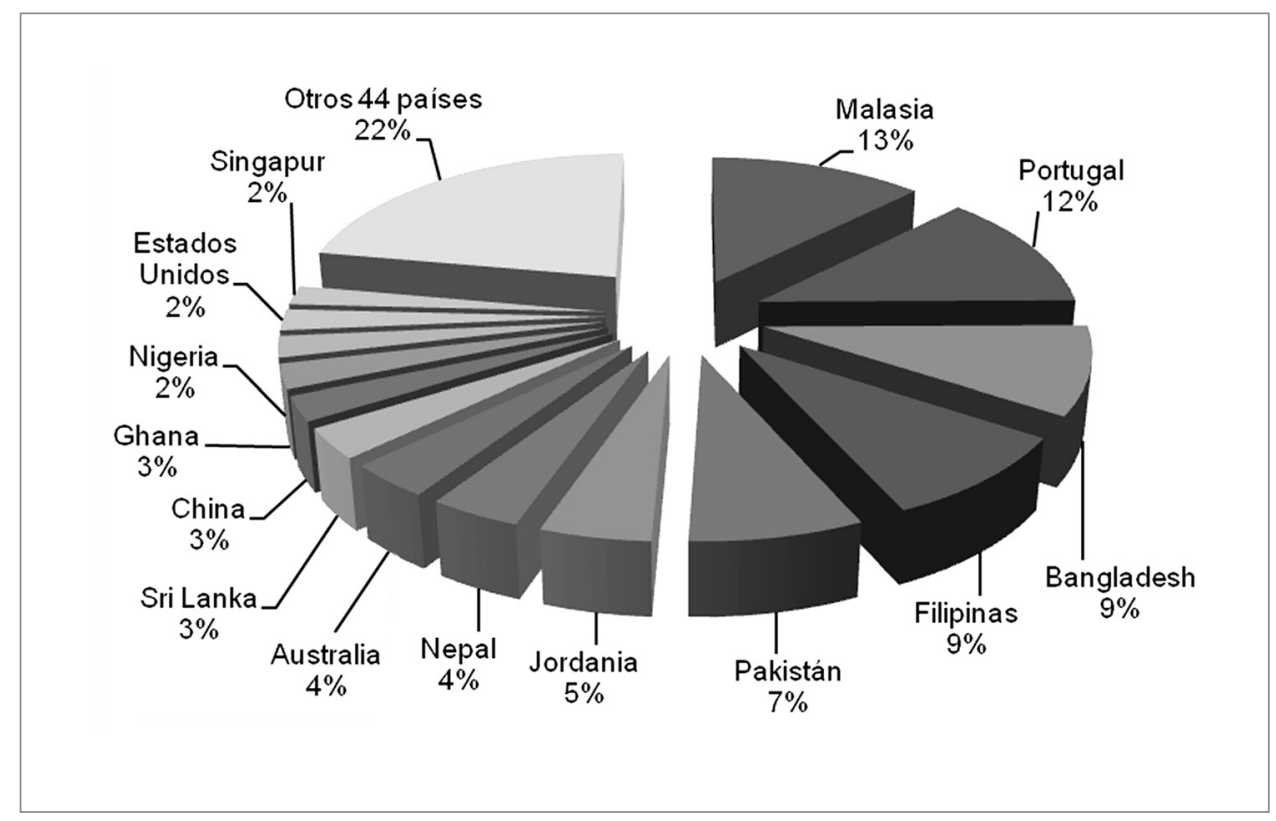

Gráfico 2: Porcentaje de personal de policía por país aportante en las diferentes misiones en Timor, 1999-2011

Fuente: elaboración propia a partir de los reportes mensuales de las NU sobre OMP

Australia lidera la fuerza multinacional de INTERFET encargada de restaurar la paz y la seguridad en Timor. Solo cinco días después de adoptada la resolución, llegaron a reunirse tropas de 22 países. Lachica (2011) argumenta que sin la rápida intervención australiana el éxito de la estabilización lograda por INTERFET no habría podido ser posible; también se debe incluir su disposición a financiar la mayor parte de los costos.

Sin embargo, este despliegue no fue repentino; previamente el primer ministro australiano, John Howard, ya se había reunido con el presidente Habibie, el 27 de abril de 1999, e instó a Indonesia a aceptar una fuerza internacional de paz antes del referéndum, pero fue rechazada (Nevins, 2005). Lachica (2011) aclara que cuando la violencia en Timor Oriental comenzó, Australia ya había demostrado su intención de liderar una fuerza multinacional con el envío de dos buques de guerra con 500 soldados a las aguas del Este de Timor.

En cuanto al personal con actividades de policía la presencia australiana no es tan destacada (gráfico2). Australia aportó 455 oficiales que lo ubican en el octavo lugar entre los mayores aportadores, tres veces menor que lo aportado por Malasia y Portugal, que lideran la presencia policial, y también superado por Bangladesh, Filipinas, Pakistán, Jordania y Nepal. 
Si bien entre los observadores y el personal de policía los aportes australianos no son significativamente superiores, su presencia sí es relevante desde el punto de vista del aporte de tropas. El porcentaje de tropas aportadas por Australia supera el $20 \%$ del total (4317), superando los porcentajes de participación de los otros 27 países aportadores (ver gráfico 3).

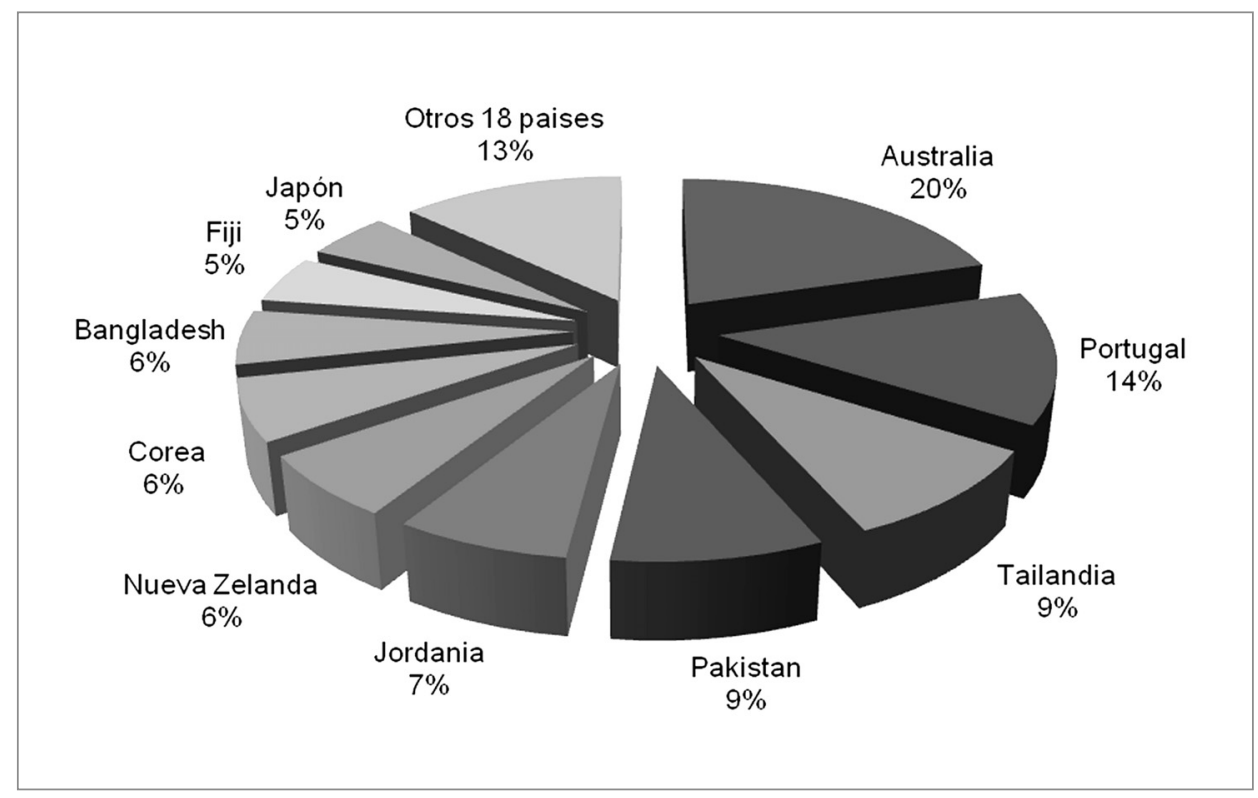

Gráfico 3: Porcentaje de tropas por país aportante en las diferentes misiones en Timor 1999-2011

Fuente: elaboración propia a partir de los reportes mensuales de la ONU sobre OMP

Más claridad arroja el análisis del comportamiento de Australia con relación a las demás operaciones de mantenimiento de paz de las Naciones Unidas y su relación con el aporte hecho a la misión en Timor Leste. Entre 2000 y 2004, periodo en el que la misión en Timor estuvo integrada por tropas militares, el 100\% de las tropas dispuestas por Australia en Operaciones de Mantenimiento de Paz se encontraban en Timor. En el personal de policía, con excepción de 2000 y 2009 (años en los que Australia no aportó personal de policía para OMP), y al 2005 (cuando fue el momento más bajo de participación, en el contexto de salida progresiva de las Naciones Unidas antes de la crisis de 2006), del total de oficiales en OMP en todo el mundo, la presencia en Timor representó entre el 50 y el 85\% (ver gráfico 4). 


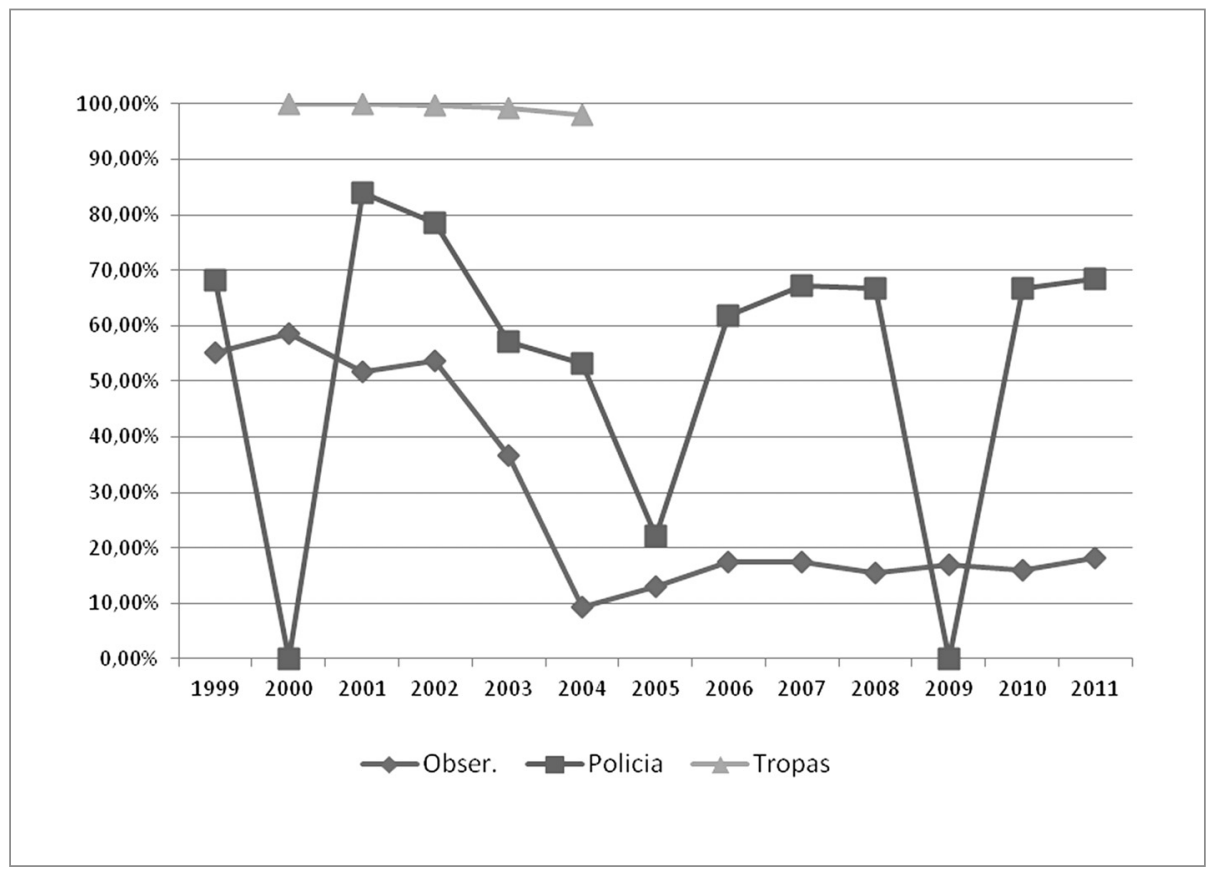

Gráfico 4: Personal de observadores, policía y tropas de Australia dispuestas en misiones en Timor con relación al personal dispuesto por Australia en el total de OPM entre 1999-2011

Fuente: elaboración propia a partir de los reportes mensuales de las NU sobre OMP

\subsection{PARTICIPACIÓN AUSTRALIANA EN LAS OPERACIONES DE MANTENIMIENTO DE PAZ COMO INSTRUMENTALIZACIÓN DE SUS INTERESES MARÍTIMOS}

Como se pudo verificar, es claro que el interés de Australia en la situación de Timor Oriental fue siempre prioritario, y varios elementos permiten deducir que bajo este apoyo existe un interés económico que impulsa tal actuación. Siguiendo a Ayoob (2002), desde el realismo subalterno, este tipo de intervención se establece como una herramienta utilizada por los estados poderosos para extender su influencia en los estados más débiles, como parece reflejar este caso en particular.

Uno de los mayores intereses que Australia tiene en Timor Leste es el relativo a la delimitación de la frontera marítima entre los dos estados en la parte correspondiente al Mar de Timor. El tema ha sido de frecuente preocupación por parte de los mandatarios australianos de manera permanente (Ayson, 2005), y su relevancia radica en las enormes reservas de petróleo y gas natural que se estiman en el lecho marino de esta área. 
Desde la década de 1980 se ha acuñado por parte de las instancias gubernamentales australianas el término de que dicho país posee al norte un $<<$ arco de inestabilidad $>>$ en su parte norte. Dentro de ese arco de inestabilidad se encuentra, de manera particular, Timor-Leste y la administración de los recursos del Timor Gap. Esta situación se profundizó en el 2006 y 2007 cuando el gobernante australiano John Howard fue objeto de críticas por parte del Partido Laborista Australiano, de estar atendiendo más preocupado de la Guerra global contra el Terror (Iraq), descuidando los asuntos domésticos, en especial, en el Suroeste del Pacífico. Así lo señaló Rudd, líder de la oposición federal para quien "si bien el Gobierno de Howard se centra en Iraq, la inestabilidad en nuestra región, nuestro barrio, nuestro propio patio trasero sigue propagándose" (Ayson, 2007, p. 215).

La controversia por los límites en el Mar de Timor se remonta a los años 70 del siglo pasado. En 1972, cuando el territorio de Timor Oriental aún estaba bajo el dominio de Portugal, Australia e Indonesia acordaron los límites marítimos entre los dos territorios. Siguiendo la fórmula de la $<<$ estructura morfológica $>>$, Australia argumenta la validez de la tesis de la prolongación natural, según la cual cada Estado ribereño debería tener derechos sobre esa parte de la plataforma continental que forma una prolongación natural de su territorio en y bajo el mar. Así, la ubicación de la frontera marítima debe ser determinada, o al menos influenciada por las características geofísicas del fondo del mar, en particular, su geología (composición y estructura) y geomorfología (forma y configuración) (Schofield, 2007).

Contrario a lo establecido en la Convención del Mar de 1958, Australia estableció mediante una ley interna la existencia de una zona adyacente hasta casi 500 metros de la Depresión de Timor (Antunes, 2002). La tesis australiana defendía que la depresión de Timor, con profundidad de más de $3.500 \mathrm{~m}$, rompe la plataforma continental entre Australia y Timor en dos espacios distintos por lo que no existe un espacio común por delimitar.

Después de acordar las fronteras marítimas con Indonesia, Australia pretendió acordar esta demarcación con Portugal. Sin embargo, Portugal no aceptó la tesis australiana que dejaba a Timor prácticamente sin plataforma continental y planteó que la frontera debía marcarse con una línea media. Con la salida de Portugal del territorio y la anexión de Indonesia, el problema de la delimitación adquirió nuevas características.

Después, bajo el dominio de Indonesia, las negociaciones iniciaron con la finalidad de complementar la territorialización del mar australiano en la zona. Desde la perspectiva australiana, el nuevo límite debía ser la continuación de lo acordado anteriormente. Sin embargo, Indonesia se negó a aceptar esta fórmula, debido a su interés de explotar la parte correspondiente al Mar de Timor. Con las nuevas condiciones planteadas por la Convención del Derecho del Mar de 1982, Indonesia afirmó su derecho a las 200 millas, apoyado por fallos de las Corte Internacional de Justicia que ratifican la irrelevancia de factores geológicos en la delimitación entre estados, contrario a la tesis de delimitación propuesta por Australia (Schofield, 2007). 
A pesar de la ventaja jurídica de Indonesia, finalmente los dos países acuerdan establecer una zona de cooperación para el desarrollo conjunto en la zona abisal del mar de Timor (Timor Gap),con asignación de derecho compartido sobre los recursos de hidrocarburos en la zona reclamada. Este acuerdo parece ser perjudicial para Indonesia, pero afirmó su posesión sobre el territorio de Timor Oriental ante cualquier reclamo posterior.

Poco más de un año después, el 22 de febrero de 1991, Portugal demandó el acuerdo ante la Corte Internacional de Justicia. Portugal argumentó que el acuerdo desconocía sus derechos de potencia administradora de Timor Oriental, bajo su consideración como territorio no autónomo; y dijo que además el acuerdo no respetaba los derechos propios de la población de Timor sobre los recursos naturales presentes en su plataforma continental.

Australia sostuvo que no existía una controversia entre ella y Portugal, que la pretensión de Portugal requería regular derechos y obligaciones de un tercer Estado no parte en los procedimientos, que Portugal no tenía capacidad para presentar el caso ante la Corte. Australia fundamenta su posición alegando la artificialidad de la demanda portuguesa, porque entiende que el verdadero sujeto de la demanda es Indonesia, que no ha sido llevado ante la Corte debido a su falta de aceptación de la jurisdicción obligatoria de la Corte (Espósito, 1996).

Ante estas circunstancias, la Corte en su sentencia Portugal v. Australia del 30 de junio de 1995, por una decisión de 14 votos a favor y dos en contra, declaró que no es competente para resolver la situación, basada en que no puede "pronunciarse sobre la legalidad de la conducta de un tercer Estado, Indonesia, sin su consentimiento, cuando la materia sobre la cual debía decidir no sólo afectaba sino que constituía el núcleo esencial para solucionar la controversia" (Espósito, 1996).

Con el proceso de independencia en 1999, se revivió el tema de la delimitación. La Convención del Derecho del Mar de 1982 ya ha sido hito definitorio para controversias de este tipo, marcando la delimitación por línea media. Sin embargo, Australia se niega a resolver la controversia por medio de la Corte Internacional de Justicia. Los líderes locales timorenses ahora reclaman áreas al este y oeste de la Zona de Cooperación, ya que los limites anteriores estaban basados en otras pequeñas islas de Indonesia (Lundahl \& Sjoholm, 2008).

Sin embargo, en ese momento la administración del territorio de Timor Oriental estaba bajo la UNTAET, que en un canje de notas establece mantener con Australia el acuerdo hasta que se terminara la transición. En 2003, ya con el primer gobierno democráticamente elegido, Timor Leste y Australia establecen la Joint Petroleum Development Area (JPDA) como continuación de la zona de cooperación (ver mapa 1). Toda el área de la JPDA estaría en el área reclamada por Timor. 
Mapa 1. Joint Petroleum Development Area (JPDA)

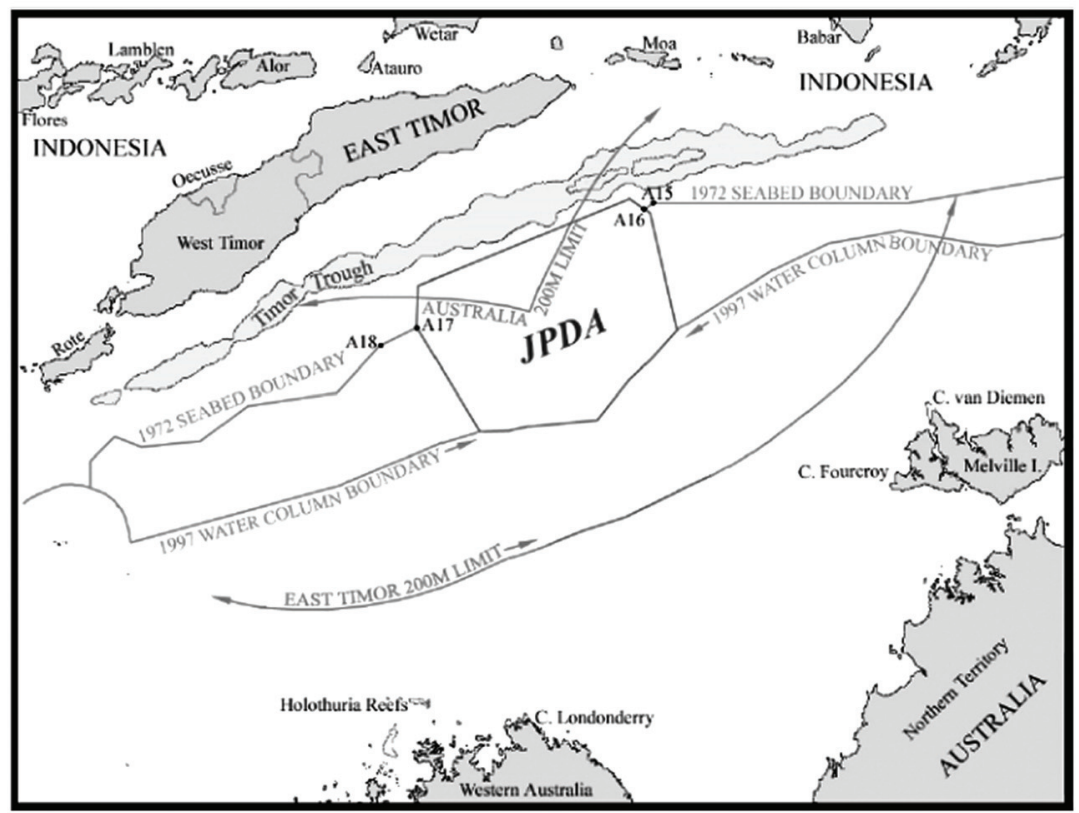

Fuente: Tomado de Antunes, N. (2002)

Para el año de 2007, con la presión de Australia, los dos estados llegan al acuerdo Treatyon Certain Maritime Arrangements in the Timor Sea (CMATS), que entró en funciones en febrero. Aunque el tratado no define la frontera marítima definitiva, prevé la distribución equitativa de los ingresos derivados del desarrollo de un complejo de gas y petróleo en el centro del Mar de Timor, y más importante aún, el aplazamiento de las reclamaciones de cada estado a la jurisdicción marítima y de fronteras en el mar de Timor durante 50 años (Schofield, 2007).

Timor-Leste acusa a Australia de continuar la explotación solitaria y ha tomado la totalidad de los ingresos de zonas limítrofes con la JPDA sobre las cuales todavía no se ha establecido un acuerdo, y de las que Timor no ha obtenido ganancias. Actualmente la totalidad de la explotación energética de Timor-Leste se concentra en la JPDA y las áreas que se suponen más ricas en recursos siguen en litigio. (Mapa 2).

No se sabe el tamaño exacto de reservas de gas y petróleo que hay en la zona en disputa. La producción de Timor se concentra en el campo de BayuUndan, en la zona de explotación compartida, donde se producen 110.000 barriles al día, zona en donde se estiman reservas de 400 millones de barriles y 3,4 billones de pies cúbicos de gas; reservas estimadas por 20 años 
Mapa 2. Petróleo en la Mar de Timor

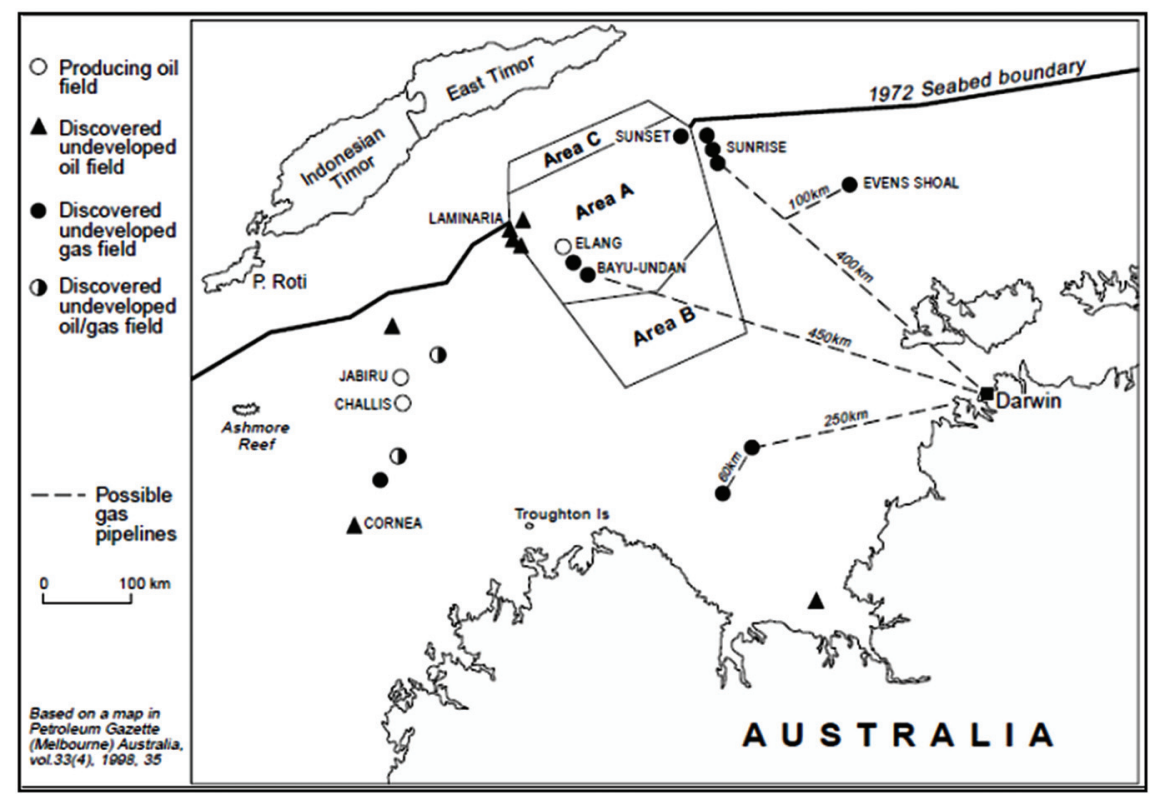

Fuente: Tomado de Prescott, V. (2000)

que oscilan entre 6 y 7 billones de dólares (Lundahl \& Sjoholm, 2008). Para el Fondo Monetario Internacional (2009) el potencial petrolero se incrementa con las nuevas exploraciones en la zona: para el 2009, las estimaciones más conservadoras apuntan a que existen reservas de 700 millones de barriles en petróleo extraíbles hasta el 2022.

Sin embargo, el potencial más grande se encuentra en el campo Sunrise, fuera de la zona de explotación compartida. El GreaterSunrise contiene un estimado entre 8.3 y 8.4 billones de pies cúbicos de gas y entre 200 y 295 millones de barriles de condensado. Bajo el acuerdo, Australia y Timor-Leste acordaron utilizar el GreaterSunrise, sobre la base de que el 20,1\% del campo se encuentra dentro del JPDA y el restante $79,9 \%$ en lo que Australia se considera titular indiscutible por estar al sur de la línea de delimitación de acuerdo con Indonesia. Por lo tanto, Timor se beneficiarían de sólo un 18,1\% de los ingresos. De manera adicional, se estima que podría haber yacimientos en tierra, pero todavía no se ha explorado (Schofield, 2007; Fondo Monetario Internacional, 2009).

Australia también defiende la idea de que el procesamiento de los recursos se realice en su territorio, lo que le generará mayores excedentes económicos. Las autoridades timorenses no están en capacidad de exigir participación en este tipo de actividades debido al limitado 
alcance de su infraestructura y a la pobreza existente en el país. Australia, gracias a su poderío y capacidad económica es el principal beneficiado del acuerdo que posterga el reclamo sobre la delimitación marítima. Sin embargo, Australia es consciente de que debe brindar al gobierno de Dili alternativas razonables de mutuo beneficio, debido a la cada vez más creciente presencia de China en la región, la cual se ha acercado de manera favorable al Estado timorense.

Para Timor-Leste, los recursos de la JPDA es una oportunidad para que esta naciente y débil nación aprenda de las negativas experiencias de otros estados que han sido maldecidos por recursos naturales, administrando los recursos en hidrocarburos de manera racional y sostenible. En este aspecto se resalta el trabajo de tesis doctoral escrito por Drysdale (2007) quien hizo una valoración cualitativa y cuantitativa de los recursos de Timor, evaluando las perspectivas estratégicas de aprovechamiento y la disposición institucional para su administración.

Una muestra de ello, es el diseño de las instituciones timorenses que tiene como piedra angular la Ley del Fondo del Petróleo, la cual fue discutida durante diez meses y cinco etapas de consultas políticas, económicas, técnicas y jurídicas. Las regalías son recibidas a través del Fondo y solo pueden ser utilizadas a partir de los presupuestos del Estado timorense. Dentro de su investigación Drysdale verificó este aspecto al aplicar entrevistas y encuestas tanto a decenas de gobernantes y tomadores de decisiones timorenses así como entre una muestra representativa de la población, donde se constata la importancia del tema en Timor-Leste:

La mayoría de los participantes (79\%) prefiere el uso de los ingresos del petróleo de Timor-Leste para financiar los gastos del presupuesto del Estado, en lugar de buscar un préstamo de una institución financiera internacional. De manera adicional, el análisis mostró que los participantes de Timor Oriental que no tienen un papel en la de toma de decisiones en la gestión de los ingresos del petróleo fueron aún más firmes en que Timor-Leste debe evitar tomar un préstamo (90\% prefirió financiar los gastos presupuestarios con los ingresos del petróleo). Hay un mandato claro de los participantes en esta investigación que la política de permanecer libre de deudas deben ser mantenida. (2007, pp. 226-227).

\section{CONCLUSIÓN}

La intervención de las Naciones Unidas en el proceso de construcción de Estado en Timor ha sido vital para garantizar la estabilidad y viabilidad en el proceso, llegando incluso a generar dependencia y problemas de viabilidad de auto-gobernabilidad y auto-sostenibilidad económica. Si bien la actualidad de Timor-Leste aún presenta un panorama lleno de complicaciones, la operación de Naciones Unidas en Timor es considerada como un ejemplo de éxito, el cual se ha convertido en un recurrente ejemplo para la reformulación modélica de las Operaciones de Mantenimiento de Paz. 
Algunas de las claves para la positiva intervención de NU en Timor Oriental fueron su capacidad de trasformación para adaptarse a las realidades de cada etapa, la implementación satisfactoria del referéndum de autodeterminación que condujo a la independencia de Timor, la instauración de garantías para procesos electorales y el trabajo conducente a la gradual reducción de los niveles de violencia. Timor se ha erigido en un laboratorio para la evolución y reconfiguración del paradigma que debe guiar las OMP, en donde se dejó atrás las tradicionales operaciones de refuerzo de la paz, para pasar a la implementación de un nuevo concepto de construcción de paz.

Sin embargo, es necesario el análisis de fondo de esta afirmación, ya que la prolongada intervención de las Naciones Unidas ha creado fuertes lazos de dependencia que imposibilitan una salida definitiva del personal, para evitar situaciones como la ocurrida en 2006 donde Timor recayó en las dinámicas de violencia interna, que pusieron en entredicho los avances en la consolidación del Estado.

En la OMP aprobadas y desarrolladas en Timor Leste, se destaca de manera singular el papel desempeñado por Australia en cuanto al liderazgo y al aporte de personal, especialmente en lo relacionado con el despliegue de tropas. Además del evidente interés humanitario, es posible encontrar que su actuación estuvo motivada por otros intereses, propios de una potencia regional que desea preservar su influencia en la zona así como la necesidad de ratificar su posición dominante en el proceso de consolidación de la territorialización de las zonas marítimas compartidas.

Bajo la administración de UNTAET, y posteriormente, bajo el primer gobierno independiente, Australia consiguió la ratificación bilateral de un tratado sobre los límites marítimos que le beneficia para acceder a los importantes recursos en hidrocarburos presentes en el Mar de Timor, y que posterga cualquier reclamo por 50 años. El débil gobierno de Timor Leste debe someterse a un acuerdo con la potencia regional, cuando la aplicación de la Convención del Mar de 1982 establece términos y derechos distintos, los cuales han sido no solo desconocidos sino que de manera directa han sido vulnerados.

Australia, por medio de la intervención de las OMP de las Naciones Unidas, coadyuvó en la estabilización interna de Timor-Leste y a la construcción de un gobierno independiente. Sin embargo, se ha tratado de un gobierno débil e influenciable que le permitió suscribir un acuerdo beneficioso sobre la delimitación y aprovechamiento del Mar de Timor. Así, dio a los inversionistas garantías políticas y legales que permitieron la explotación segura de hidrocarburos presentes en el área, y en donde la gran mayoría de las ganancias de estos procesos son para el que tiene la capacidad y la infraestructura necesaria.

Timor-Leste es un país con grandes yacimientos petroleros, pero no es una nación petrolera, ya que no tiene la infraestructura, ni los recursos económicos ni la claridad de una apropiada 
delimitación marítima. Los beneficios se están direccionando en su gran mayoría hacia otros actores como son su benefactora vecina, Australia, así como las grandes empresas corporativas petroleras involucradas en los proyectos de extracción en la Joint Petroleum Development Area (JPDA).

\section{BIBLIOGRAFÍA}

- $\quad \operatorname{AFP}(2012,24$ de Abril).Un ex guerrillero es elegido oficialmente presidente de Timor Oriental. (Consultado el 24 de abril de 2012). Recuperado de: http://www.google.com/ hostednews/afp/article/ALeqM5g9AZFegNpXqPDo2wA85Hh6IW1wUQ?docld= CNG.68566d3f23938f2193dcac775c0efee1.961

- $\quad$ Alldén, S. \& Amer, R. (2007).The United Nations and Peacekeeping: Lessons Learned from Cambodia and East Timor. Umeå Working Papers Peace and Conflict Studies, 4.

- Antunes, N. (2002). Spatial Allocation of Continental Shelf Rights in the Timor Sea: Reflections on Maritime Delimitation and Joint Development. Timor Sea Office. (Consultado el 2 de abril de 2012). Recuperado de: http://www.timorseaoffice.gov.tp/ nunoarticle13-13.pdf

- Ayoob, M. (2002). Humanitarian Intervention and State Sovereignty. The International Journal of Human Rights, 6, 1, 81-102.

- Ayson, R. (2007). The 'arc of instability' and Australia's strategic Policy. Australian Journal of International Affairs, 61, 2,215-231.

- $\quad$ Ayson, R.(2005). A shift in focus? Australia and stability in East Asia. Canberra: Australian Strategic Policy Institute.

- Boegue, V, Brown, A, Clements, K. \& Nolan, A. (2008). ¿Qué es lo "fallido"? ¿Los Estados del Sur, o la investigación y las políticas de Occidente? Un estudio sobre órdenes políticos híbridos y los Estados emergentes.(Consultado el 10 de abril de 2012). Recuperado de: http://www.berghof-handbook.net/documents/publications/spanish_boege_etal_ handbook.pdf

- $\quad$ Butler, M. (2012). Ten Years After: (Re) Assessing Neo-Trusteeship and UN State-building in Timor-Leste. International Studies Perspectives, 13, 85-104.

- $\quad$ Cadena, W. R. \& Devia, C. A. (2010). Timor-Leste o la construcción de la nación en un Estado fallido. Revista de Relaciones Internacionales, Estrategia y Seguridad. 5, 2, 39-71. 
- $\quad$ Canas, N. (2009).Multidimensional identity construction: Challenges for State-building in East Timor. En: C. Cabasset-Semedo \& F. Durand, How to build a new nation in Southeast Asia in the $21^{\text {st }}$ century? Bangkok: Irasec.

- Comissão de Acolhimento, Verdade e Reconciliação, CAVR. (2005). Chega!: (Consultado el 10 de Julio de 2010). Recuperado de: http://www.cavr-timorleste.org/().

- Drysdale, J. (2007) Sustainable development or Resource cursed? An exploration of timorleste's institutional choices (Tesis Doctoral). (Consultado el 5 de agosto de 2012). Recuperado de: https://digitalcollections.anu.edu.au/bitstream/1885/49322/13/01front.pdf

- Espósito, C. (1996). El asunto Timor Oriental ante la Corte Internacional de Justicia. Anuario de derecho internacional. XII, 617-639.

- Fondo Monetario Internacional. (2009). Democratic Republic of Timor-Leste: Selecte dissues and Statistical Appendix.(Consultado el 15 de agosto de 2012).Recuperado de: http:// www.imf.org/external/pubs/ft/scr/2009/cr09220.pdf

- $\quad$ Funaki Y. (2009). The UN and Security Sector Reform in Timor-Leste: A Widening Credibility Gap. (Consultado el 2 abril de 2012). Recuperado de:http://www.cic.nyu.edu/ peacekeeping/security_sector/docs/funaki_timor.pdf

- Group King's College. (2003).A Review of Peace Operations: A Case for Change East Timor Study Conflict, Security and Development. (Consultado el 23 de abril de 2012). Recuperado de: http://www.jsmp.minihub.org/Reports/otherresources/Peace4Timor_10_3_03.pdf

- Guardiola, N. (1989). Besar o no besar el suelo ocupado. El País. http://elpais.com/ diario/1989/10/12/internacional/624150005_850215.html.(Consultado el 22 de septiembre de 2012)

- Hicks, D. (2007). Community and nation-state in East Timor. A view from the periphery. Anthropology Today, 1, 13-16.

- Huston, R. (2011).The Australia-East Timor joint development agreement. (Consultado el 10 de abril de 2012). Recuperado de: http://www.dundee.ac.uk/cepmlp/gateway/ ?news $=31307$

- $\quad$ Kingsbury, D. \& Leach, M. (2007).East Timor: Beyond independence. Clayton: Monash University Press.

- Lachica, A. (2011). Humanitarian intervention in East Timor: An analysis of Australia's leadership role. Peace \& Conflict Review, 5, 2, 1-7. 
- $\quad$ Lundahl, M. \& Sjoholm, F. (2008). The oil resources of Timor-Leste: curse or blessing? The Pacific Review,21, 1, 67-86.

- $\quad$ Nevins, J. (2005).A not so distant horror: Mass Violence in East Timor. New York: Cornell University Press.

- $\quad$ Prescott, V. (2000). The question of East Timor's maritime boundaries. IBRUBoundary and Security Bulletin, 7, 4, 72-82.

- $\quad$ Rocha, A. y Morales, D. E. (2010). Potencias medias y potencias regionales en el Sistema Político Internacional: dos modelos teóricos. Geopolítica(s.), 1, 2, 251-279.

- $\quad$ Schofield, C. (2007). Minding the Gap: The Australia-East Timor Treaty on Certain Maritime Arrangements in the Timor Sea (CMATS).The International Journal Of Marine And Coastal Law, 22, 2, 189-234.

- $\quad$ Simonsen, S. (Oct. 2008). Identidad desde la violencia: la nueva división este-oeste en Timor Oriental. Revista Académica de Relaciones Internacionales, GERI-UAM. (Consultado el 1 de octubre de 2012). Recuperado de :http://www.relacionesinternacionales.info/ ojs/article/view/126.html

- Smith, M. (2003).Peacekeeping in East Timor: the path to independence. London: Lynne Reinner.

- Taylor, J. G. (2001).The emergence of nationalism movement in East Timor. En Kratoska, P. (Ed.), South East. Colonial history. Londres: Routhledge.

- United Nation Peacekeeping (2012). Contributions by country.1999, 2000, 2001, 2002, 2003, 2004, 2005, 2006, 207, 2008, 2009, 2010, 2011. (Consultado el 8 de abril de 2012). Recuperado de:http://www.un.org/en/peacekeeping/resources/statistics/ contributors.shtml

- United Nation Peacekeeping (2012). Country contributions detailed by misión. 1999, 2000, 2001, 2002, 2003, 2004, 2005, 2006, 207, 2008, 2009, 2010, 2011.(Consultado el 8 de abril de 2012). Recuperado de:http://www.un.org/en/peacekeeping/resources/statistics/ contributors.shtml

- United Nation Peacekeeping (2012). UNMIT. (Consultado el 22 de abril de 2012). Recuperado de: http://www.un.org/en/peacekeeping/missions/unmit/

- Zurbriggen. C. (2005). Política exterior, defensa y las operaciones de paz: iuna estrategia coherente? El caso de Uruguay. Revista Fuerzas Armadas y Sociedad. 19, 1, 85-109. 